\title{
Hipoglicemia recurrente como causa reversible de síndrome demencial en adultos mayores diabéticos, a propósito de un caso
}

\author{
ANDRÉS AIZMAN, LUIS MANUEL SANHUEZA A. ${ }^{\mathrm{a}}$, \\ MIRENTXU IRURETAGOYENA ${ }^{\mathrm{b}}$, EDUARDO ABBOTT, \\ JUAN CARLOS RODRÍGUEZ, LUIS ROJAS
}

\section{Cognitive impairment probably related to repeated episodes of hypoglycemia. Report of one case}

We report a 78 year-old diabetic woman, treated with gliburide and met-formin, consulting in the emergency room for a non fluctuating impairment in consciousness. She had a history of similar episodes in the last two months. A brain CAT scan showed an old putamen lacunar infarction. Noteworthy was the presence of a low glycosilated hemoglobin level of 5.2\%. Hypoglycemic medications were discontinued and the patient was discharged in good conditions. After six months of follow up, the patient did not have further episodes of impairment of consciousness.

(Rev Med Chile 2010; 138: 1285-1287).

Key words: Cognitive dissonance; Diabetes mellitus; Hyoglycemia.

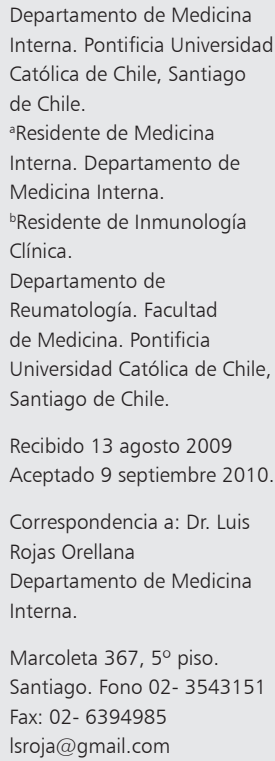

L a demencia es particularmente prevalente en la población de 65 años o más, afectando en Estados Unidos a 5-10\% de este grupo, duplicándose cada 5 años hasta alcanzar 30 a 40\% a los 80 años.

Menos de $10 \%$ de las causas de demencia se consideran reversibles. Entre estas condiciones se cuentan alteraciones tiroideas, déficit de vitamina B12, hidrocéfalo normotensivo, hematoma subdural crónico, neurosífilis, VIH y desórdenes psiquiátricos como la pseudodemencia depresiva ${ }^{1}$.

Una causa poco reconocida es el deterioro cognitivo producido por hipoglicemias recurrentes. Presentamos un caso clínico que destaca la importancia de reconocer dicho diagnóstico, especialmente considerando los objetivos de metas terapéuticas estrictas preconizados por las guías actuales y por la potencial reversión de los sín- tomas y la prevención de daño cognitivo crónico que implicaría su oportuno diagnóstico y manejo.

\section{Caso clínico}

Mujer de 78 años, autovalente, independiente en actividades de la vida diaria, con escolaridad completa, tiene antecedentes de hipertensión arterial, hipotiroidismo, hipoacusia y diabetes mellitus tipo 2 (DM 2) manejada con glibenclamida $10 \mathrm{mg}$ y metformina $1.700 \mathrm{mg}$ al día, con lo cual lograba glicemias capilares adecuadas.

Fue llevada por familiares al Servicio de Urgencia, para evaluación por compromiso cualitativo de conciencia, no fluctuante, de más de 24 hrs de evolución, sin signos de focalización y con recuperación espontánea completa. Su familia señaló 
la ocurrencia cada vez más frecuente de episodios similares en los últimos 2 meses, indicando además, alteraciones de la memoria reciente, de la conducta y alteraciones autolimitadas de la marcha, sin trastorno del control de esfínteres, con repercusión funcional por pérdida parcial de su autovalencia.

Ingresó vigil, orientada témporo-espacialmente, atenta, hemodinámicamente estable, afebril, sin apremio respiratorio y con un examen neurológico en el que sólo destacó un rendimiento anormal en el cuestionario Mini Mental, obteniendo 22 puntos, cuyo resultado sugiere la posibilidad de deterioro cognitivo (puntaje esperado para nivel de escolaridad $>24$ puntos). Entre sus exámenes de laboratorio destacaron glicemia, creatininemia, natremia, calcemia, TSH, niveles de vitamina B12 y hemograma, normales. No presentó elevación de parámetros inflamatorios, ELISA VIH negativo y RPR no reactivo. Se realizó una tomografía axial computada de cerebro no contrastada, que pesquisó un infarto lacunar hemorrágico putaminal izquierdo, probablemente antiguo. El electroencefalograma mostró lentitud intermitente generalizada. Eco-Doppler carotideo evidenció ateromatosis carotídea derecha sin compromiso hemodinámico. Ninguno de estos hallazgos logró explicar el cuadro clínico.

Se manejó con régimen alimentario diabético, sin hipoglicemiantes orales, evolucionando en forma asintomática y con glicemias en rango adecuado. La hemoglobina glicosilada A1C fue de $5,2 \%$, lo que hizo sospechar la posibilidad de episodios de hipoglicemia recurrentes. Por ello, se decidió suspender glibenclamida y disminuir la dosis de metformina previo al alta.

En controles ambulatorios, transcurridos dos y luego seis meses post alta, la paciente se encontraba vigil, orientada témporo-espacialmente, bien conectada al medio, logrando 26 puntos en Mini Mental en ambas ocasiones. Su familia no refirió nuevos episodios de alteración de conciencia, ni conductuales, interpretándose el cuadro original como secundario a hipoglicemias subclínicas frecuentes.

\section{Discusión}

Como ilustra este caso, una de las condiciones que puede producir deterioro cognitivo en adultos mayores y simular un síndrome demencial es la hipoglicemia recurrente, fenómeno posible de observar en pacientes con diabetes mellitus $(\mathrm{DM})^{2}$, en especial cuando son tratados en forma estricta persiguiendo objetivos de hemoglobina glicosilada $\mathrm{A} 1 \mathrm{C}<7 \%{ }^{3}$. Es por esto que se plantea considerar objetivos menos estrictos en este subgrupo de pacientes, y se sugiere hemoglobina glicosilada A1C entre 7 y $8 \%$, aunque actualmente no está definida la meta ideal ${ }^{3,4}$.

Recientemente, investigaciones en modelos animales han encontrado relación entre hipoglicemia crónica o recurrente y deterioro cognitivo reversible ${ }^{5}$. Sin embargo, no suele incluirse en el proceso de diagnóstico de causas reversibles de síndrome demencial. Esto es de especial relevancia ya que el envejecimiento normal se asocia a retardo en el reconocimiento sintomático de la hipoglicemia, observándose en ancianos una menor probabilidad de experimentar síntomas de alerta previo al compromiso cognitivo relacionado con ésta ${ }^{6}$.

Se ha observado que el suministro de glucosa a las áreas de alta demanda es más difícil en el cerebro envejecido, debido a una marcada reducción de la difusión de la glucosa en el líquido extracelular cerebral ${ }^{7}$. Así, el envejecimiento disminuye la capacidad de aumentar la glucosa circulante en respuesta a catecolaminas y con ello la capacidad de aumentar el suministro para la función cognitiva en situaciones de estrés ${ }^{8}$. Este efecto del envejecimiento normal, aumentaría el riesgo de hipoglicemia severa en pacientes añosos tratados con sulfonilureas o insulina?.

Se ha evidenciado en modelos animales que la hipoglicemia prolongada induciría un aumento del transporte de glucosa a través de la barrera hemato-encefálica (BHE) por medio de un fenómeno de up regulation de la expresión de GLUT 1 en la superficie luminal de la BHE y de GLUT 3 en las neuronas ${ }^{10-13}$.

Existen estudios en humanos que son consistentes con este fenómeno. Así, se produciría un aumento de la tolerancia a glicemias anormalmente bajas por la optimización de las concentraciones de glucosa intersticial en el cerebro, retardando la aparición de síntomas neuroglucopénicos de alerta $^{14}$, lo que podría llevar a un retardo en el diagnóstico de hipoglicemia y producir diversos grados de deterioro cognitivo (alteraciones conductuales y/o cognitivas subagudas) ${ }^{15}$.

Además de lo ya descrito, la hipoglicemia recurrente genera una falla en el aumento de los 
niveles de adrenalina circulantes que normalmente siguen a la caída de la glicemia, constituyendo un síndrome clínico denominado hipoglicemia asociada a falla autonómica ${ }^{15,16}$.

Estas observaciones, sumadas a los recientes reportes de aumento en la mortalidad de diabéticos tipo 2 manejados bajo esquemas terapéuticos orientados al logro de niveles de $\mathrm{Hb}$ glicosilada A1C $<6 \%{ }^{17}$, sugieren considerar una mayor flexibilidad en las metas de control metabólico, especialmente en el subgrupo de adultos mayores ${ }^{18}$.

A su vez, parece relevante considerar e incorporar esta nueva entidad dentro de los diagnósticos diferenciales de causas potencialmente reversibles de síndrome demencial.

\section{Referencias}

1. Hoyl T, Aravena C. Demencias. En: Geriatría y Genitología Marín PP, Gac H, Carrasco M, editores. Santiago, Chile. Ediciones Universidad Católica de Chile; 2006. p. 187-91.

2. Gregg EW, Engelgau MM, Narayan V. Complications of diabetes in elderly people. BMJ 2002; 325: 916-7.

3. American Diabetes Association. Standards of medical care in diabetes. Diabetes Care 2010; 33: S11-S61.

4. Brown AF, Mangione CM, Saliba D, Sarkisian CA. Guidelines for improving the care of the older person with diabetes mellitus. J Am Geriatr Soc 2003; 51: S265-80.

5. Mcnay EC. The impact of recurrent hypoglycemia on cognitive function in aging. Neurobiol Aging 2005; 26 Suppl 1: 76-9.

6. Turnheim K. Drug therapy in the elderly. Exp Gerontol 2004; 39: 1731-8

7. Sykova E. Diffusion properties of the brain in health and disease. Neurochem Int 2004; 45: 453-66.
8. Mabry TR, Gold PE, Mccarty R. Age-related changes in plasma catecholamine and glucose responses of F-344 rats to a single foot-shock as used in inhibitory avoidance training. Neurobiol Learn Mem 1995; 64: 146-55.

9. Matyka K, Evans M, Lomas J, Cranston I, Macdonald I, Amiel SA. Altered hierarchy of protective responses against severe hypoglycemia in normal aging in healthy men. Diabetes Care 1997; 20: 135-41.

10. Mcnay EC, Sherwin RS. Effect of recurrent hypoglycemia on spatial cognition and cognitive metabolism in normal and diabetic rats. Diabetes 2004; 53: 418-25.

11. Mccall AL, Du Z, Javorsky B. Acute but not chronic hypoglycemia downregulates brain MAP kinase phosphorylation. Diabetes 2005; 54: A69.

12. Uehara Y, Nipper V, Mccall AL. Chronic insulin hypoglycemia induces GLUT-3 protein in rat brain neurons. Am J Physiol Endocrinol Metab 1997; 272: E716-9.

13. Simpson IA, Vannucci SJ. Glucose transport into brain: effects of hypoglycemia. Diabetes Nutr Metab 2002; 15: 281-4.

14. Mccall AL. Altered glycemia and brain-update and potential relevance to the aging brain. Neurobiol Aging 2005; 26 Suppl 1: 70-5.

15. Cryer PE. Hypoglycemia-associated autonomic failure in diabetes. Am J Physiol Endocrinol Metab 2001; 281: E1115-21.

16. Mcnay EC, Williamson A, Mccrimmon RJ, Sherwin RS. Cognitive and neural hippocampal effects og long-term moderate recurrent hypoglycemia. Diabetes 2006; 55 : 1088-95.

17. Members of the Action to Control Cardiovascular Risk in Diabetes (Accord) Study Group. Effects of intensive glucose lowering in type 2 diabetes. N Engl J Med 2008; 358: 2545-59.

18. Bremer JP, Jauch-Chara K, Hallschmid M, Schmid S, Schultes B. Hypoglycemia unawareness in older compared with middle-aged patients with type 2 diabetes. Diabetes Care 2009; 32: 1513-7. 